\title{
Rotational Self-motion Cues Improve Spatial Learning when Teleporting in Virtual Environments
}

\author{
Alex F. Lim \\ Iowa State University \\ aflim@iastate.edu \\ Lucia A. Cherep \\ Iowa State University \\ lacherep@iastate.edu
}

\author{
Jonathan W. Kelly \\ Iowa State University \\ jonkelly@iastate.edu \\ Grace C. Freed \\ Iowa State University \\ gfreed@iastate.edu
}

\author{
Nathan C. Sepich \\ Iowa State University \\ nsepich@iastate.edu
Stephen B. Gilbert
Iowa State University
gilbert@iastate.edu


Figure 1: Screenshots showing the participant's perspective when using the partially concordant teleporting interface (left panel) and the discordant teleporting interface (right panel). Also shown is the list of objects indicating whether each object has or has not been visited yet.

\begin{abstract}
Teleporting interfaces are widely used in virtual reality applications to explore large virtual environments. When teleporting, the user indicates the intended location in the virtual environment and is instantly transported, typically without self-motion cues. This project explored the cost of teleporting on the acquisition of survey knowledge (i.e., a "cognitive map"). Two teleporting interfaces were compared, one with and one without visual and body-based rotational self-motion cues. Both interfaces lacked translational selfmotion cues. Participants used one of the two teleporting interfaces to find and study the locations of six objects scattered throughout a large virtual environment. After learning, participants completed two measures of cognitive map fidelity: an object-to-object pointing task and a map drawing task. The results indicate superior spatial learning when rotational self-motion cues were available. Therefore, virtual reality developers should strongly consider the benefits of rotational self-motion cues when creating and choosing locomotion interfaces.
\end{abstract}

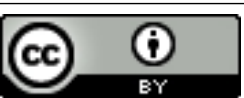

This work is licensed under a Creative Commons Attribution International 4.0 License.

SUI '20, October 31-November 1, 2020, Virtual Event, Canada

(c) 2020 Copyright held by the owner/author(s).

ACM ISBN 978-1-4503-7943-4/20/10.

https://doi.org/10.1145/3385959.3418443

\section{CCS CONCEPTS}

- Applied computing $\rightarrow$ Psychology; $\bullet$ Human-centered computing $\rightarrow$ Virtual reality.

\section{KEYWORDS}

virtual reality, spatial navigation, survey knowledge, teleporting

\section{ACM Reference Format:}

Alex F. Lim, Jonathan W. Kelly, Nathan C. Sepich, Lucia A. Cherep, Grace C. Freed, and Stephen B. Gilbert. 2020. Rotational Self-motion Cues Improve Spatial Learning when Teleporting in Virtual Environments. In Symposium on Spatial User Interaction (SUI '20), October 31-November 1, 2020, Virtual Event, Canada. ACM, New York, NY, USA, 7 pages. https://doi.org/10.1145/ 3385959.3418443

\section{INTRODUCTION}

A key feature of modern, immersive virtual reality (VR) is the ability to explore virtual environments (VEs) by physically walking to translate (i.e., change position) and physically turning to rotate (i.e., change orientation). However, this experience in VR is often limited by the walkable tracked space (e.g., the user's living room is bounded by obstacles including furniture and walls). The challenge of exploring VEs that exceed the size of the tracked space is typically resolved by introducing a locomotion interface, such as joystick navigation or teleportation. The focus of the current study is on the teleporting interface and their effects on acquiring survey 
knowledge in a large-scale VE which is central for executing novel detours or pointing to unseen landmarks.

Teleporting has become a popular locomotion interface, whereby the user points to a location in the VE and is discretely teleported to that location, typically without any accompanying self-motion cues. Teleporting overcomes physical space constraints, but this interface lacks translational self-motion cues which indicate a change in position, and can also lack rotational self-motion cues which indicate a change in orientation. The advantages of the teleporting interface are that it is easy to use $[1,16]$ and reduces cybersickness $[6,16,20,33]$ compared with other interfaces that include visual self-motion (e.g., using a gamepad or joystick to locomote). On the other hand, there are disadvantages with teleporting stemming primarily from the lack of body-based cues associated with real walking, which are vital for keeping track of self-location during travel (i.e., spatial updating).

As described in section 1.2, there is strong evidence that translational and rotational self-motion cues are critical for successful navigation $[15,25]$ and that teleporting negatively affects spatial orientation $[3,14]$. The main contribution of this study is to evaluate the impact of rotational self-motion cues when teleporting on the accuracy of acquired survey knowledge (i.e., a cognitive map). The results have applied implications for VR locomotion interfaces as well as basic implications for spatial cognitive theory.

\subsection{Concordance framework for locomotion interfaces}

When walking in VR, movement through the VE is concordant with movement of the body. Within this concordance framework [3] natural walking provides all self-motion cues, including proprioceptive cues, vestibular cues, visual cues, and efferent motor commands (idiothetic information) that are essential for spatial updating (i.e., keeping track of self-location within the environment). However, VEs often exceed the limited tracked physical space, and therefore require different VR locomotion interfaces to overcome this limitation. Because locomotion interfaces are designed to separate the user's movement through the virtual environment from their movement through the real environment, these locomotion interfaces typically compromise the concordance between movement through the VE and movement of the body.

Teleporting is a relatively new, but now widely adopted locomotion interface for navigating large VEs. In the most common implementation of the teleporting interface, the user physically rotates their body to turn in the VE but teleports to translate (i.e., to change location). In this case, rotations include all body-based and visual self-motion cues that normally occur when rotating in the real world, but translation includes no self-motion cues. Within the concordance framework, this teleporting interface is considered partially concordant (see Figure 2, left panel) because rotational movement through the VE is concordant with rotating the body, but translational movement is discordant with movement of the body.

Discordant teleporting - a different variety of the teleporting interface - is a locomotion interface that enables the user to teleport to translate and to rotate, with no associated body movement (see Figure 2, right panel). To translate and rotate, the user positions and orients a marker (e.g., an arrow) on the ground plane and is then teleported to that location and orientation. Advantages of discordant teleporting interface include accessibility that is necessary for users who have impaired or otherwise limited mobility (e.g., confined to a wheelchair, or in a confined space as with in-flight entertainment) preventing them from easily using concordant or partially concordant locomotion interfaces. In the context of the concordance framework, movement through the VE is discordant with movement of the body. Joystick or other gamepad control devices that provide smooth visual movement without accompanying body movement are another popular example of discordant interfaces. However, those methods often cause cybersickness in VR [6] because of the mismatch between visual and body-based self-motion cues.

There is increasing evidence that teleporting negatively affects spatial orientation. In a triangle completion task (travel two outbound path legs before pointing back to an unseen path origin), absolute pointing errors were higher when using partially concordant teleporting compared to walking, and highest when using discordant teleporting compared to partially concordant teleporting $[3,14]$. However, it is unknown whether teleporting negatively impacts the acquisition of survey knowledge for large-scale VEs.

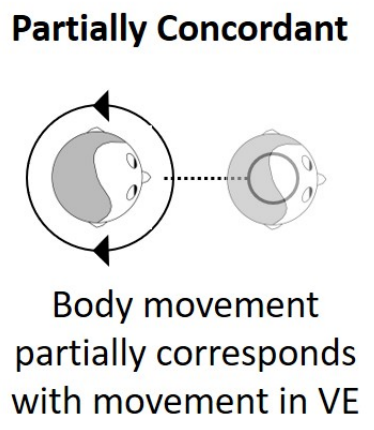
Discordant

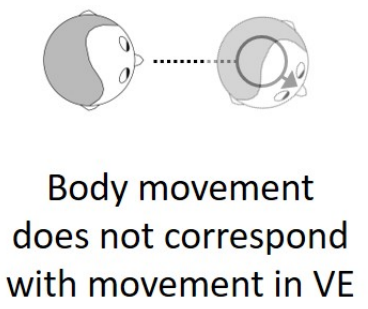

Figure 2: The two locomotion interfaces tested in this study. When using the partially concordant teleporting interface (left panel), the user rotates their body to rotate in the VE but teleports to translate. When using the discordant teleporting interface (right panel), the user teleports to rotate and translate.

\subsection{Research on self-motion cues and survey knowledge acquisition}

Survey knowledge, sometimes referred to as a "cognitive map," is the mental representation of distances and directions between multiple points in an environment [28, 34]. Survey knowledge is useful for complex spatial behaviors, such as generating novel shortcuts or taking detours. Despite the relatively clear evidence that self-motion cues are critical to spatial orientation, research on the importance of self-motion cues to survey knowledge acquisition is surprisingly equivocal.

In small spaces, in which all or most of the space is visible from a single vantage point (termed "vista spaces" [21]), survey knowledge can be acquired through visual scanning and studying of the 
surrounding environment. Vista spaces therefore do not require significant locomotion to fully apprehend. Larger spaces, which cannot be viewed in their entirety from a single vantage point (termed "environmental spaces" [21]), require locomotion in order to experience and learn the full environment. One popular theory of spatial learning is that survey knowledge of an environmental space involves linking together knowledge of multiple vista spaces. Importantly, those links between vista spaces depend on successful spatial updating during locomotion, which provides the glue that creates a single representation of the larger environmental space $[22,24]$. One theory [32] goes even farther to claim that spatial updating is the primary input into survey learning of environmental spaces. Although spatial updating is error prone over medium or long travel distances, the theory is that remembered visual scenes are used to reset accumulated error in the spatial updating system.

Given the theorized importance of spatial updating for survey learning, it stands to reason that manipulations which negatively impact spatial updating (e.g., removal of body-based self-motion cues) should also negatively impact survey knowledge. However, there is surprising disagreement in the literature on this topic, with some studies finding that body-based cues facilitate survey learning $[11,26,27,30]$ and others showing no benefit of such cues $[13,17,19,31]$. Even studies reporting a benefit of body-based cues for survey learning have reported a relatively small benefit $[2,5,29,30]$, which stands in contrast to the large benefit of bodybased cues in spatial updating tasks like triangle completion [3, 14], and further calls into question whether spatial updating plays a central role in the acquisition of survey knowledge. Therefore, it is not clear whether the lack of self-motion cues when teleporting would negatively impact survey learning.

\subsection{Current experiment and hypotheses}

Hypotheses and experimental details were pre-registered prior to data collection on the Open Science Framework: https://osf.io/vpfja/. Participants learned object locations in a large VE using one of two teleporting interfaces: partially concordant teleporting or discordant teleporting. The interfaces differed in availability of rotational self-motion cues. After learning, survey knowledge was assessed using the scene and orientation-dependent pointing (SOP) task $[8,35]$, in which the participant was placed at the location of one object and asked to point to the unseen location of another object. Absolute pointing error in the SOP task is commonly used to measure the fidelity of survey knowledge. Survey knowledge was also assessed by asking participants to draw a map of the environment.

Given the large impact of rotational self-motion cues on spatial updating [3, 14], it was hypothesized that learning the VE with the discordant teleporting interface would lead to less accurate survey knowledge compared to learning with the partially concordant teleporting interface. Therefore, we predicted that discordant teleporting would lead to larger absolute errors in the SOP task and lower accuracy in the map drawing task compared to partially concordant teleporting.

Sample size was estimated by conducting a power analysis ( $G$ Power) with the following parameters: independent samples t-test between two groups, one-tailed test, Cohen's $d$ effect size, $d=.50$ (medium effect size), alpha level $=.05$, minimum Power needed to detect an effect $=.80$. Effect size was estimated from a related study [12] that compared survey knowledge accuracy after learning through in an HMD with rotational self-motion cues versus learning in desktop VR, and reported a medium effect size Cohen's $d=.44$.

\section{METHOD}

\subsection{Participants}

A total of 118 students participated in exchange for credit in an undergraduate psychology course, and of those, 11 were removed in the final analysis for the following reasons: two indicated they had prior knowledge of the VE from playing the video game CounterStrike, six were due to technical issues, one ended early after reporting cybersickness, one had difficulty seeing clearly, and one failed to follow instructions. Of the remaining 107 participants, 51 were assigned to partially concordant teleporting (28 women, 23 men) and 56 were assigned to discordant teleporting (32 women, 24 men).

\subsection{Hardware and software}

The equipment consisted of an HTC Vive head mounted display (HMD), which presented stereoscopic images at $1080 \times 1200$ resolution per eye, refreshed at $90 \mathrm{~Hz}$ with $100^{\circ}$ horizontal and $110^{\circ}$ vertical binocular FOV. Graphics were rendered on a Windows 10 computer with an Intel Corei7-9700K processor and Nvidia GeForce RTX 2080 graphics card using Unity 3D software. Head position and orientation were tracked using the Lighthouse tracking system. One wireless handheld controller was used by participants to control the teleporting interfaces and to perform the SOP task.

\subsection{Stimuli}

The VE (see overhead map in Figure 3, and perspective views in Figure 1 and Figure 4) was imported from the first-person shooter game Counter-Strike (Valve Corporation) into Unity (Unity Technologies) and the version used in this study was developed by vrchris. The use and reproduction of this asset is allowed under Creative Commons license Attribution 4.0 International (CC BY 4.0).

The VE was multi-level, with two floors accessible by stairs. The VE was modified by introducing six new objects (bikes, rubber duck, car, flowers, lion statue, robot) and by restricting access so that participants could not travel inside of buildings.

\subsection{Design}

In a between-participant design, participants were assigned to use the partially concordant teleporting interface or the discordant teleporting interface to learn the locations of six objects within the VE. Learning was constrained to seven minutes. After learning, participants performed the SOP task testing knowledge of interobject relationships and drew a sketch map of the environment. The primary dependent measure was absolute pointing error on the SOP task. Sketch map accuracy was also evaluated.

\subsection{Teleporting interfaces}

When using the partially concordant teleporting interface, participants physically turned their body to rotate and teleported to 




Figure 3: Bird's-eye view of the VE and object locations. Participants were never exposed to this map. The green highlighted area shows the second floor of the environment; all other areas were on the first floor. Italy VE map adapted from the video game Counter-strike (Valve Corporation).

translate. When using the discordant teleporting interface, participants teleported to translate and rotate. A virtual replica of the handheld controller was always visible, and its position and orientation were linked to that of the actual controller held by the participant. The partially concordant teleporting interface was controlled by positioning a white circle with surrounding white ring in the intended location on the ground plane (Figure 1, left panel). A thin red line extended from the controller to the center of the white circle. The participant pressed and held the trackpad located on the top of the controller while using the controller to manipulate the location of the teleport marker. Releasing the trackpad teleported the participant to the selected location (orientation was unchanged). The discordant teleporting interface was controlled by positioning and orienting a magenta ring with an arrow on one side (Figure 1, right panel). A thin red line extended from the joystick to the center of the ring. The participant pressed and held the trackpad button to bring up the teleporting ring, and rotated the ring by moving the thumb around the edge of the circular trackpad. Releasing the trackpad button teleported the participant to the selected location and orientation.

\subsection{Measures}

For each SOP trial (Figure 4), participants were placed at the location of one object (i.e., they viewed the virtual environment from that location and could freely turn to look in any direction) and were instructed to point in the direction of another object (e.g., "You are now standing at the Lion, please point the laser at the Duck."). Participants completed 30 trials, representing every combination of objects. Trials were blocked by standing location (i.e., all trials while standing at the lion were completed in one block). Block order was randomized, as was trial order within each block.

Average absolute error was calculated for each participant by averaging across all 30 trials. This was the primary measure of survey knowledge accuracy. Response time was also analyzed to evaluate whether differences in pointing error between interfaces were due to speed-accuracy trade-off. However, beyond this usage of response time data, no a priori predictions were made about response time differences across the two interfaces because response time seemed more likely to be related to confidence than to survey knowledge accuracy.

Sketch maps of the learned environment were analyzed using a bidimensional regression with a map drawing software [10], which computes an $\mathrm{R}^{2}$ value reflecting the correlation between object locations on the participant's sketch map and object locations on the true map [9]. Other map features beyond object locations, such as corridor walls, were not included in the map analysis. Bidimensional regression allows map scale and map orientation to vary freely, which is important in the current study because participants were unconstrained in the orientation and scale of their map (except for the restriction that they draw their map on a 8.5 " by 11 " paper).

\subsection{Procedure}

After providing informed consent, the participant was fitted with the HMD and provided training by traveling around in a impoverished VE until they felt comfortable with the assigned teleporting interface. All participants stood in one fixed location in the middle of the laboratory surrounded by four small bumpers placed at their feet to prevent stepping away from the standing location while rotating in the partially concordant teleporting interface.

The experimenter explained to the participant that they would have seven minutes to explore the VE and learn the locations of six objects in no particular order. The participant was also instructed to remember the relative directions between objects and that they would later be tested on this information. All participants began exploring at the start location marked in Figure 3. The list of object names was accessible to the participant at any time by holding the Vive controller within their field of view (see Figure 1). Once an object was discovered, the participant tagged it by pointing at it and pulling the trigger with the Vive controller, causing a visual update to the list indicating remaining objects to search for. If all objects were discovered with time remaining, the participant was instructed to continue exploring until time expired. If the participant did not find all six objects in the allotted time, the experimenter verbally guided the participant to the unvisited object(s). 


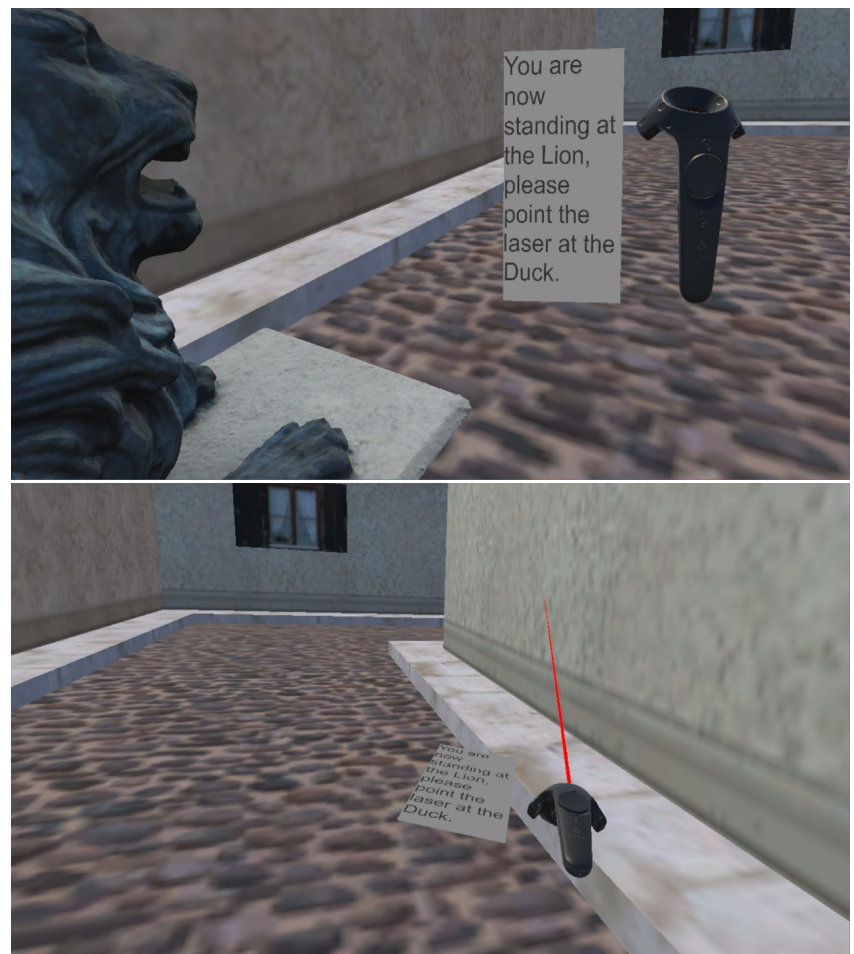

Figure 4: Screenshots showing the participant's perspective when performing the SOP task. Instructions for each pointing trial were always available next to the Vive controller (top panel), and a virtual laser attached to the Vive controller was used to indicate the direction of the pointing response (bottom panel).

After learning, the participant remained in the HMD to perform the SOP task. After completing the SOP task, the participant removed the HMD and was instructed to draw a bird's-eye view sketch map of the VE they experienced including any paths, buildings, and all objects. The participant was provided with a pencil and paper, along with a list of the object names to ensure that all objects were placed on the sketch map. The study took approximately 30 minutes to complete.

\section{RESULTS}

Nineteen participants were unable to find all six objects on their own within the time limit. This occurred more frequently with the discordant teleporting $(\mathrm{N}=15)$ interface compared to the partially concordant interface $(\mathrm{N}=4)$. The conclusions are identical regardless of whether the analyses include those participants who did not find all objects in the allotted time. The results presented here include all participants.

Absolute angular errors and sketch map scores were not normally distributed, as assessed by Shapiro-Wilk's test $(p<.05)$. However, Welch's t-test is robust to deviation from normality [7]. Assumptions of homogeneity of variance were not violated, as assessed using Levene's Test $(p>.05)$ with equal variances assumed between the two conditions [23].

\subsection{SOP task}

Absolute angular error on the SOP task is shown in the left panel of Figure 5. It was hypothesized that errors would be lower after learning with the partially concordant teleporting interface compared to the discordant teleporting interface.

Welch's independent samples t-test revealed a statistically significant difference between the two interfaces: pointing error was lower for partially concordant teleporting $(M=39.49, S D=21.52)$ compared to discordant teleporting $(M=55.25, S D=22.17), t(104.56)$ $=3.73, p<.001$, Cohen's $d=.72$. Pointing error in both conditions was well below 90 degrees, which represents chance performance.

Pointing response time (in seconds) was analyzed to evaluate whether the pointing error results were influenced by speed-accuracy trade-off. This was evaluated both within and between participants, and neither analysis showed evidence of speed-accuracy trade-off. The within-participant correlation between absolute error and response time was significantly positive $(M=0.07, S E=0.02), t(106)$ $=3.38, p<.001$. This indicates that, at the level of the individual participant, trials with larger pointing error also resulted in longer response time. Pointing response time was also analyzed using Welch's independent samples t-test in order to compare across interfaces (this was a between-participant comparison). There was no significant difference between pointing response time after learning with the partially concordant teleporting interface $(M=6.2, S E=$ $.32)$ and the discordant teleporting interface $(M=6.8, S E=.27)$, $t(100.24)=1.42, p=.159$, Cohen's $d=.28$.

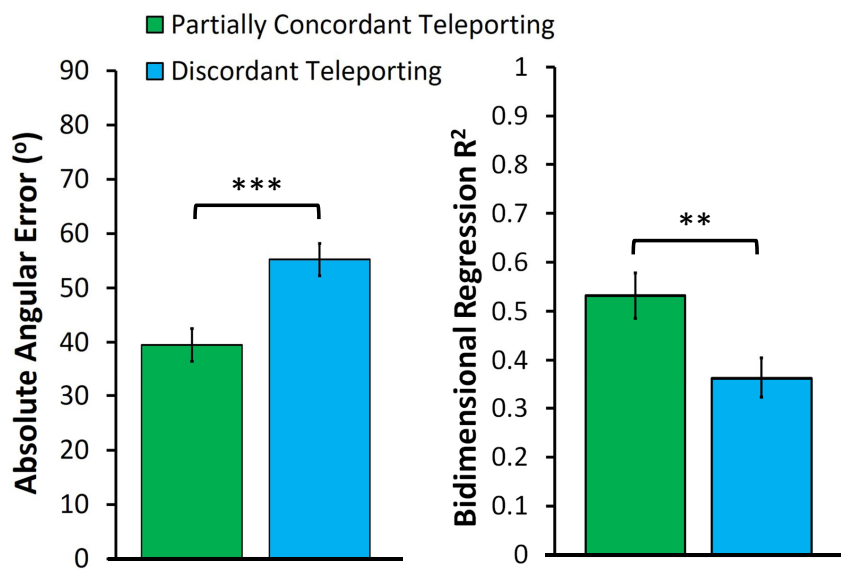

Figure 5: Left: Average absolute angular error (in degrees) on the SOP task. Right: $\mathrm{R}^{2}$ values calculated from bidimensional regression using sketch maps. Error bars represent \pm 1 standard error. ${ }^{*} p<.05,{ }^{* *} p<.01,{ }^{* * *} p<.001$

\subsection{Sketch maps}

Bidimensional regressions calculated from the sketch maps are shown in the right panel of Figure 5. It was hypothesized that sketch maps would be less accurate when learning occurred with the discordant teleporting interface compared to the partially concordant teleporting interface. Map drawing accuracy was analyzed by comparing average $\mathrm{R}^{2}$ values calculated from bidimensional regression between the two teleporting interfaces. 
Welch's independent samples t-test was conducted and a significant difference was found: participants who used the partially concordant teleporting condition produced maps that were more accurate $(M=.53, S D=.33)$ compared to map drawings from participants who used the discordant teleporting interface $(M=.36$, $S D=.30), t(101.02)=3.95, p=.008$, Cohen's $d=.53$.



Figure 6: Examples of two sketch maps that differ in accuracy. All sketch maps were quantitatively compared to the target map (Figure 3). The top panel shows a sketch map with very high accuracy $\left(R^{2}=.94\right)$. The bottom panel shows a sketch map with very low accuracy $\left(R^{2}=.04\right)$. The example sketch maps are from two different participants who explored the VE using partially concordant teleporting. Note that the sketch map analysis only considered object locations and not other map features, such as corridors.

\section{DISCUSSION}

Previous research indicates rotational self-motion cues are important to spatial updating [15], leading to better triangle completion performance with partially concordant teleporting compared to discordant teleporting $[3,14]$. However, there is disagreement in the literature as to whether self-motion cues during learning enhance the accuracy of acquired survey knowledge [11, 13, 17, 19, 26, 27, 30, 31]. The current project clearly demonstrates that rotational self-motion cues during exploration are important to developing accurate survey knowledge of a large-scale VE. These conclusions were supported by two classic measures of survey knowledge: object-toobject pointing performance and sketch maps.

Some participants were unable to locate all six objects within the fixed learning time, and were therefore guided by the experimenter to each remaining object. This occurred more frequently when using the discordant teleporting interface compared to the partially concordant teleporting interface, which highlights the importance of rotational self-motion cues. However, conclusions drawn from analyses of the SOP task and the sketch maps are unaffected by excluding participants who failed to locate all six objects within the time limit.

There are at least two possible explanations for why availability of rotational self-motion cues affected survey knowledge accuracy. First, manipulation of rotational self-motion cues most likely affected spatial updating [3], which in turn may have affected survey knowledge acquisition. We believe this is the most likely connection between our manipulation and survey knowledge acquisition. However, this explanation hinges on the theory that spatial updating is central survey knowledge acquisition [22, 24, 32], and this theory needs further evaluation. Second, manipulation of rotational selfmotion cues may have affected the usability of the interface, which negatively affected learning, perhaps by affecting cognitive load [18]. Participants received training with their assigned interface, but brief training might not be sufficient for equating the two interfaces in terms of usability. Individual differences in performance may be one way to evaluate whether the first explanation holds merit: individuals who are better at spatial updating [4] should also, therefore, form more accurate cognitive maps. We plan to test this relationship in future work.

Future work should compare performance when teleporting with a full walking condition in which all self-motion cues are present, in order to evaluate whether translational self-motion cues are important to survey learning. Physical space constraints in the current study prevented inclusion of such a walking condition.

The environment used in the current experiment was somewhat complex, with restricted sight lines and several intersecting corridors. Future work should evaluate whether these results generalize to different types of VEs, including VEs that vary in complexity.

This research should encourage VR designers to consider the spatial cognitive costs associated with teleporting interfaces. Teleporting provides the user with greater convenience and flexibility, but these advantages come at the expense of spatial learning.

\section{ACKNOWLEDGMENTS}

Pre-registration, videos, data, supplemental analyses, and links to experiment code are available on the Open Science Framework: 
https://osf.io/vpfja/. This material is based upon work supported by the National Science Foundation under Grant Number CHS1816029.

\section{REFERENCES}

[1] E. Bozgeyikli, A. Raij, S. Katkoori, and R. Dubey. 2016. Point and teleport locomotion technique for virtual reality.. In Proc. Annual Symposium on ComputerHuman Interaction in Play (Austin, TX). ACM, New York City, NY, 205-216. https://doi.org/10.1145/2967934.2968105

[2] S. S. Chance, F. Gaunet, A. C. Beall, and J. M. Loomis. 1998. Locomotion mode affects the updating of objects encountered during travel: The contribution of vestibular and proprioceptive inputs to path integration. Presence: Teleoperators and Virtual Environments 7 (1998), 168-178. https://doi.org/10.1162/ 105474698565659

[3] L. A. Cherep, A. F. Lim, J. W. Kelly, D. Acharya, A. Velasco, E. Bustamante, A. G. Ostrander, and S. B. Gilbert. 2020. Spatial cognitive implications of teleporting through virtual environments. fournal of Experimental Psychology: Applied (2020). https://doi.org/10.1037/xap0000263

[4] L. A. Cherep, A. F. Lim, J. W. Kelly, A. Miller, and S. B. Gilbert. 2020. Individual differences in teleporting through virtual environments: A latent profile analysis. In 2020 IEEE Conference on Virtual Reality and 3D User Interfaces Abstracts and Workshops (VRW). 724-725. https://doi.org/10.1109/VRW50115.2020.00213

[5] E. R. Chrastil and W. H. Warren. 2012. Active and passive contributions to spatial learning. Psychonomic Bulletin and Review 19 (2012), 1-23. https://doi.org/10. 3758/s13423-011-0182-x

[6] C. G. Christou and P. Aristidou. 2017. Steering versus teleport locomotion for head mounted displays. In Augmented Reality, Virtual Reality, and Computer Graphics, L. De Paolis, P. Bourdot, and A. Mongelli (Eds.). Lecture Notes in Computer Science, Vol. 10325. Springer-Verlag, London, 431-446. https://doi org/10.1007/978-3-319-60928-7 37

[7] M. Delacre, D. Lakens, and C. Leyes. 2017. Why psychologists should by default use Welch's t-test instead of Student's t-test. International Review of Social Psychology 30, 1 (2017). https://doi.org/10.5334/irsp.82

[8] A.D. Ekstrom, A. EGF. Arnold, and G. Iaria. 2014. A critical review of the allocentric spatial representation and its neural underpinnings: toward a networkbased perspective. Frontiers in human neuroscience 8 (2014), 803. https //doi.org/10.3389/fnhum.2014.00803

[9] A. Friedman and B. Kohler. 2003. Bidimensional regression: Assessing the configural similarity and accuracy of cognitive maps and other two-dimensional data sets. Psychological methods 8, 4 (2003), 468. https://doi.org/10.1037/1082-989X.8.4.468

[10] A. L. Gardony, H. A. Taylor, and T. T. Brunyé. 2016. Map Drawing Analyzer Software for quantitative analysis of sketch maps. Behavior research methods 48 , 1 (2016), 151-177. https://doi.org/10.3758/s13428-014-0556-x

[11] S. C. Grant and L. E. Magee. 1998. Contributions of proprioception to navigation in virtual environments. Human Factors 40, 8 (1998), 489-497. https://doi.org/10. $1518 / 001872098779591296$

[12] Q. He, T. P. McNamara, B. Bodenheimer, and A. Klippel. 2019. Acquisition and transfer of spatial knowledge during wayfinding. Fournal of Experimental Psychology: Learning, Memory, and Cognition 45, 8 (2019), 1364. https://doi.org/ 10.1037/xlm0000654

[13] D. J. Huffman and A. D. Ekstrom. 2019. A modality-independent network underlies the retrieval of large-scale spatial environments in the human brain. Neuron 104, 3 (2019), 611-622. https://doi.org/10.1016/j.neuron.2019.08.012

[14] J. W. Kelly, A. G. Ostrander, A. F. Lim, L. A. Cherep, and S. B. Gilbert. 2020 Teleporting through virtual environments: Effects of path scale and environment scale on spatial updating. IEEE Transactions on Visualization and Computer Graphics 26, 5 (2020), 1841-1850. https://doi.org/10.1109/TVCG.2020.2973051

[15] R. L. Klatzky, J. M. Loomis, A. C. Beall, S. S. Chance, and R. G. Golledge. 1998 Spatial updating of self-position and orientation during real, imagined, and virtual locomotion. Psychological Science 9 (1998), 293-298. https://doi.org/10.1111/14679280.00058

[16] E. Langbehn, P. Lubos, and F. Steincke. 2018. Evaluation of locomotion techniques for roomscale VR: Joystick, teleportation, and redirected walking.. In Proc. Virtual Reality International Conference (Laval, France). ACM, New York City, NY, Article 4, 9 pages. https://doi.org/10.1145/3234253.3234291

[17] H. Li and N. A. Giudice. 2013. The effects of immersion and body-based rotation on learning multi-level indoor virtual environments.. In Proceedings of the Fifth ACM SIGSPATIAL International Workshop on Indoor Spatial Awareness (Orlando, Florida). ACM, New York, NY, USA, 8-15.

[18] W. E. Marsh, J. W. Kelly, V. J. Dark, and J. H. Oliver. 2013. Cognitive demands of semi-natural virtual locomotion. Presence: Teleoperators and Virtual Environments 22, 3 (2013), 216-234. https://doi.org/10.1162/PRES_a_00152

[19] E. Mellet, L. Laou, L. Petit, L. Zago, B. Mazoyer, and N. Tzourio-Mazoyer. 2010 Impact of the virtual reality on the neural representation of an environment Human brain mapping 31, 7 (2010), 1065-1075. https://doi.org/10.1002/hbm.20917
[20] K. R. Moghadam, C. Banigan, and E. D. Ragan. 2018. Scene transitions and teleportation in virtual reality and the implications for spatial awareness and sickness. IEEE Transactions on Visualization and Computer Graphics (2018). https: //doi.org/10.1109/TVCG.2018.2884468

[21] D. R. Montello. 1993. Scale and multiple psychologies of space. In Spatial Information Theory A Theoretical Basis for GIS, A. U. Frank and I. Campari (Eds.). Lecture Notes in Computer Science, Vol. 716. Springer, Berlin, Heidelberg, Berlin, 312-321. https://doi.org/10.1007/3-540-57207-4_21

[22] D. R. Montello and H. L. Pick Jr. 1993. Integrating knowledge of vertically aligned large-scale spaces. Environment and Behavior 25, 3 (1993), 457-484. https://doi.org/10.1177/0013916593253002

[23] B. K. Moser and G. R. Stevens. 1992. Homogeneity of variance in the two-sample means test. The American Statistician 46, 1 (1992), 19-21. https://doi.org/10.1080/ 00031305.1992.10475839

[24] A. E. Richardson, D. R. Montello, and M. Hegarty. 1999. Spatial knowledge acquisition from maps and from navigation in real and virtual environments. Memory and Cognition 27, 4 (1999), 741-750. https://doi.org/10.3758/BF03211566

[25] R. A. Ruddle and S. Lessels. 1998. For efficient navigational search, humans require full physical movement, but not a rich visual scene. Psychological Science 17, 6 (1998), 460-465. https://doi.org/10.1111/j.1467-9280.2006.01728.x

[26] R. A. Ruddle, E. Volkova, and H. H. Bülthoff. 2011. Walking improves your cognitive map in environments that are large-scale and large in extent. $A C M$ Transactions on Computer-Human Interaction (TOCHI) 18, 2 (2011), 1-20. https: //doi.org/10.1145/1970378.1970384

[27] R. A. Ruddle, E. Volkova, B. Mohler, and H. H. Bülthoff. 2011. The effect of landmark and body-based sensory information on route knowledge. Memory and Cognition 39, 4 (2011), 686-699. https://doi.org/10.3758/s13421-010-0054-z

[28] A. W. Siegel and S. H. White. 1975. The development of spatial representations of large-scale environments. Advances in child development and behavior 10, 4 (1975), 9-55. https://doi.org/10.1016/S0065-2407(08)60007-5

[29] D. Waller and N. Greenauer. 2007. The role of body-based sensory information in the acquisition of enduring spatial representations. Psychological research 71 , 3 (2007), 322-332. https://doi.org/10.1007/s00426-006-0087-x

[30] D. Waller, J. M. Loomis, and D. B. Haun. 2004. Body-based senses enhance knowledge of directions in large-scale environments. Psychonomic Bulletin and Review 11, 1 (2004), 157-163. https://doi.org/10.3758/BF03206476

[31] D. Waller, J. M. Loomis, and S. D. Steck. 2003. Inertial cues do not enhance knowledge of environmental layout. Psychonomic Bulletin and Review 10, 4 (2003), 987-993. https://doi.org/10.3758/BF03196563

[32] R. F. Wang. 2016. Building a cognitive map by assembling multiple path integration systems. Psychonomic Bulletin and Review 23, 3 (2016), 692-702. https://doi.org/10.3758/s13423-015-0952-y

[33] T. Weissker, A. Kunert, B. Fröhlich, and A. Kulik. 2018. Spatial updating and simulator sickness during steering and jumping in immersive virtual environments.. In Proc. IEEE Conference on Virtual Reality and 3d User Interfaces (VR) (Reutlingen, Germany). IEEE, Washington, D.C., 97-104. https://doi.org/10.1109/ VR.2018.8446620

[34] T. Wolbers and C. Büchel. 2005. Dissociable retrosplenial and hippocampal contributions to successful formation of survey representations. Fournal of Neuroscience 25, 13 (2005), 3333-3340. https://doi.org/10.1523/JNEUROSCI.470504.2005

[35] H. Zhang, K. Zherdeva, and A.D. Ekstrom. 2014. Different "routes" to a cognitive map: dissociable forms of spatial knowledge derived from route and cartographic map learning. Memory and Cognition 42 (2014), 1106-1117. https://doi.org/10. 3758/s13421-014-0418-x 